\title{
Compressible Gas Flow Inside a Two Dimensional Uniform Microchannel
}

\author{
Chunpei Cai* and Danny D. Liu ${ }^{\dagger}$ \\ ZONA Technology Inc., Scottsdale, Arizona 85258 \\ Iain D. Boyd ${ }^{\ddagger}$ \\ University of Michigan, Ann Arbor, Michigan, 48109
}

\begin{abstract}
This paper analyzes a compressible gas flow through a microchannel, and reports a complete set of first order analytical solutions. This work represents an extension of the previous work of Arkilic on compressible flows through a microchannel. First, by comparing the magnitudes of different forces in the compressible gas flow, we obtain proper estimations for the Reynolds and Mach numbers at the channel exit. Second, based on these estimations, we obtain asymptotic analytical solutions of velocities, pressure and temperature distributions of compressible gas flow inside the microchannel with a relaxation of the isothermal assumption, which was previously used by many researchers. Numerical simulations of compressible flows through microchannels with a compressible Navier-Stokes equation solver are performed, combined with velocity slip and temperature jump wall boundary conditions. The numerical simulation results validate the analytical results from this study.
\end{abstract}

\section{Nomenclature}

$\epsilon$

$u, v$

$H, L$

height to length ratio, $H / L$

specific heat ratio

Boltzmann constant, or thermal conductivity coefficient

mean free path

gas viscosity

number density

pressure

velocity

$K n$

microchannel height and length

\footnotetext{
${ }^{*}$ CFD Specialist, 9489 E. Ironwood Square Drive, previously graduate student research assistant at the University of Michigan, AIAA Member.

†President, Professor Emeritus Arizona State University, 9489 E. Ironwood Square Drive, AIAA Fellow.

$\ddagger$ Professor, Department of Aerospace Engineering, 1320 Beal Ave., AIAA Associate Fellow.
}

$$
1 \text { of } 14
$$

American Institute of Aeronautics and Astronautics 


\begin{tabular}{ll}
$M a$ & Mach number, $=u_{o} / \sqrt{\gamma R T_{o}}$ \\
$\rho$ & density \\
$R$ & universal gas constant \\
$R e$ & Reynolds number, $=\rho_{o} u_{o} H / \mu$ \\
$P$ & ratio between inlet and outlet pressure, $p_{i} / p_{o}$ \\
$P r$ & Prandtl number \\
$Q$ & mass flow rate \\
$\sigma_{u}$ & momentum accommodation coefficient \\
$\sigma_{T}$ & energy accommodation coefficient \\
$T$ & temperature \\
$\Theta_{u}$ & coefficient, $\left(2-\sigma_{u}\right) / \sigma_{u}$ \\
$\Theta_{T}$ & coefficient, $\left(2-\sigma_{T}\right) / \sigma_{T}$ \\
subscript & \\
$i, o$ & averaged inlet and outlet property \\
$w$ & wall property \\
superscript & \\
\hline & nondimensional property
\end{tabular}

\section{Introduction}

ICROCHANNELS are important components for many Micro-Electro-Mechanical Systems(MEMS), and it has been an interesting research topic to study the gas flows in microchannels. In the literature, there are many discussions about gas flows in tubes. ${ }^{1,2,3}$ Due to the small scale, many unexpected phenomena have been observed. For example, the experimental measurements by Pong et $a l^{4}$ and Arkilic et $a l^{5,6}$ showed that the pressure distribution inside a microchannel is not linear, and there is velocity slip on the channel wall. It is well accepted that for flows through microchanels, the Navier-stokes equations are still applicable if a slip wall boundary condition is used, and the analytical solutions for the flow distributions along the channel were obtained by many researchers with an isothermal assumption, for example, the work by Arkilic et $a l,{ }^{6}$ Karniadakis and Beskok, ${ }^{7}$ and Zohar et al. ${ }^{8}$ Numerically, there were many simulations of compressible flows in a microchannel as well, for example, with the direct simulation Monte Carlo method, ${ }^{9,10,11}$ the Information Preservation method, ${ }^{12}$ the direct solving Boltzmann method, ${ }^{13}$ the Boltzmann equations ${ }^{14}$ and gas-kinetic BGK-Burnett method. ${ }^{15}$ Discussions of thermal heating are reported as well. ${ }^{16,17}$

The work in this paper is based on Arkilic's previous classical work ${ }^{6}$ which is extended here to quasiisothermal flow conditions, with proper determination of the flow parameters. We include the temperature effects by further considering the energy equation. The purpose to consider the temperature distribution is based on the following fact: Microchannels usually have a tiny dimension of height, typically in the order of micrometers, the heat conduction can be quite significant because of the large temperature gradients, even with a small temperature variation inside the channel. Hence, a study of the temperature field inside the microchannel, as an extension of Arkilic's previous classical work may be of practical importance.

\section{2 of 14}

American Institute of Aeronautics and Astronautics 
In this paper, we first perform an order estimation for the Reynolds and Mach numbers at the channel exit, based on the fact that the momentum change along the flow direction, the viscous force and the pressure drop must be balanced everywhere in the flow field. Further, without the isothermal assumption, a set of asymptotic solutions of velocities, pressure and temperature for the compressible gas flow in a microchannel is obtained. Finally, to validate several important analytical conclusions from this study, numerical simulations of compressible gas flows inside a microchannel are performed with a Navier-Stokes equation solver and proper boundary conditions.

\section{Problem Description and Governing Equations}

Suppose a microchannel has a height of $H$ and a length of $L$, and the average compressible outflow gas properties of pressure, density, temperature, velocity and number density are $p_{o}, \rho_{o}, T_{o}, U_{o}, n_{o}$. The inlet pressure is several times larger than the outlet pressure, and the temperature in the flowfield permits small variations, i.e, following a "quasi-isothermal" condition, instead of the exact isothermal condition. The averaged outlet quantities are used to normalize the following governing equations and boundary conditions.

The full set of two-dimensional Navier-Stokes equations for steady, compressible gas flows are: ${ }^{18}$

$$
\begin{gathered}
\frac{\partial(\rho u)}{\partial x}+\frac{\partial(\rho v)}{\partial y}=0 \\
\rho u \frac{\partial u}{\partial x}+\rho v \frac{\partial u}{\partial y}=-\frac{\partial p}{\partial x}+\mu\left(\frac{\partial^{2} u}{\partial x^{2}}+\frac{\partial^{2} u}{\partial y^{2}}+\frac{1}{3}\left(\frac{\partial^{2} u}{\partial x^{2}}+\frac{\partial^{2} v}{\partial x \partial y}\right)\right) \\
\rho u \frac{\partial v}{\partial x}+\rho v \frac{\partial v}{\partial y}=-\frac{\partial p}{\partial y}+\mu\left(\frac{\partial^{2} v}{\partial x^{2}}+\frac{\partial^{2} v}{\partial y^{2}}+\frac{1}{3}\left(\frac{\partial^{2} v}{\partial y^{2}}+\frac{\partial^{2} u}{\partial x \partial y}\right)\right) \\
\rho u C_{p} \frac{\partial T}{\partial x}+\rho v C_{p} \frac{\partial T}{\partial y}=u \frac{\partial p}{\partial x}+v \frac{\partial p}{\partial y}+k\left(\frac{\partial^{2} T}{\partial x^{2}}+\frac{\partial^{2} T}{\partial y^{2}}\right)+\mu\left[2\left(\frac{\partial u}{\partial x}\right)^{2}+2\left(\frac{\partial v}{\partial y}\right)^{2}+\left(\frac{\partial v}{\partial x}+\frac{\partial u}{\partial y}\right)^{2}-\frac{2}{3}\left(\frac{\partial u}{\partial x}+\frac{\partial v}{\partial y}\right)^{2}\right] \\
p=\rho R T=n k T
\end{gathered}
$$

With a consideration of the velocity slip and temperature jump on the top and bottom walls, the boundary conditions are: ${ }^{19}$

$$
\begin{aligned}
& u_{w}=\mp \frac{2-\sigma_{u}}{\sigma_{u}} \lambda\left(\frac{d u}{d y}\right)_{w}+\frac{3}{4} \frac{\mu}{\rho T_{w}}\left(\frac{\partial T}{\partial x}\right)_{w}, y= \pm H / 2 \\
& T-T_{w}=\mp \frac{2-\sigma_{T}}{\sigma_{T}} \frac{2 \gamma}{\operatorname{Pr}(\gamma+1)} \lambda\left(\frac{\partial T}{\partial y}\right)_{w}, y= \pm H / 2
\end{aligned}
$$

\section{Order Estimations for Reynolds and Mach Numbers at Channel Exit}

Take a small slice of flow field as illustrated by Figure 1, based on the momentum equation along the $\mathrm{x}$-direction, the viscous force, pressure drop and momentum change through this small control volume must be balanced with the following relation:

$$
\frac{H}{2} \frac{\delta p}{\delta x} \delta x+\frac{H}{2} \rho_{o} u_{o} \frac{\delta u}{\delta x} \delta x \simeq \mu\left(\frac{\partial u}{\partial y}\right)_{w} \delta x
$$

With linear approximations, the gradient terms can be expressed as:

$$
\frac{\delta p}{\delta x} \sim \frac{p_{o}-p_{i}}{L}, \frac{\delta u}{\delta x} \sim \frac{u_{o}-u_{i}}{L}, \frac{\delta u}{\delta y} \sim \frac{u_{o}-u_{w}}{H} \sim \frac{u_{o}}{H},
$$

where ${ }_{i, o}, w$ represent quantities at inlet, outlet and wall, respectively. Note in the last approximation, we assume that $u_{w} \ll u_{o}$, and the velocity along the $\mathrm{x}$-direction has a profile close to a linear relation. Hence,

$$
3 \text { of } 14
$$

American Institute of Aeronautics and Astronautics 
the above relations are more appropriate in a laminar flow situation rather than that for a turbulent flow. This laminar flow condition requires a limitation for the Reynolds number which is defined with the outlet flow properties and the channel height: $R e=\rho_{o} U_{o} H / \mu<300$.

With the above relations, and $u_{i}^{2}=u_{o}^{2}\left(p_{o} / p_{i}\right)^{2} \ll u_{o}^{2}$, Equation 8 can be simplified:

$$
O\left(\frac{p_{o}-p_{i}}{L} H\right) \sim O\left(\rho_{o} u_{o}^{2} \frac{H}{L}\right)+O\left(\mu \frac{u_{o}}{H}\right)
$$

or

$$
O\left((P-1) \epsilon M a^{-2}\right) \sim O(\gamma \epsilon)+O\left(\frac{\gamma}{R e}\right)
$$

where $\epsilon$ is the ratio of channel height to channel length. For microchannels, the $\epsilon$ value varies from $10^{-1}$ to $10^{-5}$.

There are four possibilities among the three terms in the above equation: two terms are significantly greater than the other term and hence these two terms balance each other, or all of the three terms share the same order.

1. The pressure drop term balances with the convection term while the viscous term is smaller. This leads to $(P-1) \epsilon M a^{-2} \sim \gamma \epsilon, \gamma / R e=O\left(\gamma \epsilon^{2}\right)$, or $\gamma / R e=O\left(\gamma \epsilon^{3}\right)$. The following final relations are obtained: $M a \sim O(1), R e \sim O\left(\epsilon^{-2}\right)$ or $\operatorname{Re} \sim\left(\epsilon^{-3}\right)$. Due to the fact that a flow instability usually happens at Reynolds number greater than 300 for microchannels, for these two situations, the flow speed in the microchannel is high enough to reach a turbulent flow state. These cases are less realistic and they invalidate the linear assumption for the velocity profiles.

2. The pressure drop term balances with the viscous term while the convection term is small. This condition leads to $(P-1) \epsilon M a^{-2} \sim \gamma / R e, \gamma / R e=O(1)$ or $\gamma / R e=O\left(\epsilon^{-1}\right)$ or $\gamma / R e=O\left(\epsilon^{-2}\right)$. The above relations yield the following results: $M a \sim O\left(\epsilon^{1 / 2}\right), R e \sim O(1)$; or $M a \sim O(\epsilon), R e \sim O(\epsilon)$; or $M a \sim\left(\epsilon^{3 / 2}\right), R e \sim O\left(\epsilon^{2}\right)$. From these results, it is evident that the assumption $M a \sim O(\epsilon)$ and $R e \sim O(\epsilon)$, which was adopted by Arkilic, is a special case from this category.

3. The convection term balances with the viscous term, and the pressure drop term is smaller. This condition leads to $\gamma / R e=\gamma \epsilon,(P-1) \epsilon M a^{-2} \sim \gamma \epsilon^{2}$, or $(P-1) \epsilon M^{-2} \sim \gamma \epsilon^{3}$. These relations yield the following results: $\operatorname{Re} \sim O\left(\epsilon^{-1}\right), M a \sim O\left(\epsilon^{-1 / 2}\right)$ or $M a \sim O\left(\epsilon^{-1}\right)$. These cases involve supersonic flow and are to be neglected in our discussion.

4. All of the three terms are of the same order: $(P-1) \epsilon M a^{-2} \sim \gamma \epsilon \sim \gamma / R e$, and the final results are: $M a \sim O(1), R e \sim O\left(\epsilon^{-1}\right)$.

For compressible flows inside microchannels without any electric/magnetic field effects, the pressure drop is the only force to drive the flow down the channel, and the viscous force on the wall is the only force to slow down the flow, hence, they are equally important. It is appropriate to neglect the convection term due to the slow velocity. With a small $H / L$ ratio and a typical value greater than 2 for the inlet-outlet pressure ratio, $P$, Cases 1, 3 and 4 are supersonic flows or even hypersonic flows that are not physically possible inside microchannels without electric/magnetic effects. Further consider the fact that the combination of $M a \sim O\left(\epsilon^{3 / 2}\right)$ and $R e \sim O\left(\epsilon^{2}\right)$ represents an extremely slow gas flow inside a microchannel, we further neglect this situation as well. Two groups of parameters from Case Two are selected to start the derivation process for the analytical solutions. Further with the gaskinetic theory for the Knudsen number, $K n=\sqrt{\pi \gamma / 2}(M a / R e)$, these two cases are summarized as following:

1. Case A: $M a \sim O\left(\epsilon^{1 / 2}\right), R e \sim O(1), K n \sim O\left(\epsilon^{1 / 2}\right)$;

2. Case B: $M a \sim O(\epsilon), R e \sim O(\epsilon), K n \sim O(1)$. 
For both cases, the viscous friction force and the pressure drop are of the same leading order, and for both cases the following general, important relation holds:

$$
M a^{2} / R e=O\left(\frac{(P-1) \epsilon}{\gamma}\right)
$$

\section{Asymptotic Solutions}

By following the steps in Arkilic's work ${ }^{6}$ we normalize the flow properties with the averaged values at the channel outlet, $\mathrm{x}, \mathrm{y}$ coordinates with the channel length and height, $L$ and $H$, and use ' to denote the non-dimensional quantities. We further assume the following formats for the non-dimensional quantities: ${ }^{6}$

$$
\begin{aligned}
& u^{\prime}=u_{1}+\epsilon u_{2}+\epsilon^{2} u_{3}+\ldots, v^{\prime}=v_{1}+\epsilon v_{2}+\epsilon^{2} v_{3}+\ldots, \\
& p^{\prime}=p_{1}+\epsilon p_{2}+\epsilon^{2} p_{3}+\ldots, \rho^{\prime}=\rho_{1}+\epsilon \rho_{2}+\epsilon^{2} \rho_{3}+\ldots, \\
& n^{\prime}=n_{1}+\epsilon n_{2}+\epsilon^{2} n_{3}+\ldots, T^{\prime}=T_{1}+\epsilon T_{2} .
\end{aligned}
$$

Note an expansion for the temperature is assumed, which will lead to an analytical result for the temperature distribution.

For the flowfield in a microchannel, the pressure and density change rapidly through the flowfield while the temperature actually does not change significantly. Based on this fact, it is reasonable to assume a quasi-isothermal condition with $T_{1}=1$. The goal of this section is to obtain the analytical results for $u_{1}$, $v_{2}, p_{1}, \rho_{1}, T_{2}$ and the mass flow rate $Q$.

The nondimensional governing equations are:

$$
\begin{gathered}
\epsilon \frac{\partial\left(\rho^{\prime} u^{\prime}\right)}{\partial x^{\prime}}+\frac{\partial\left(\rho^{\prime} v^{\prime}\right)}{\partial y^{\prime}}=0 \\
\epsilon \rho^{\prime} u^{\prime} \frac{\partial u^{\prime}}{\partial x^{\prime}}+\rho^{\prime} v^{\prime} \frac{\partial u^{\prime}}{\partial y^{\prime}}=-\frac{\epsilon}{\gamma M^{2}} \frac{\partial p^{\prime}}{\partial x^{\prime}}+\frac{1}{R e}\left(\epsilon^{2} \frac{\partial^{2} u^{\prime}}{\partial x^{\prime 2}}+\frac{\partial^{2} u^{\prime}}{\partial y^{\prime 2}}+\frac{1}{3}\left(\epsilon^{2} \frac{\partial^{2} u^{\prime}}{\partial x^{\prime 2}}+\epsilon \frac{\partial^{2} v^{\prime}}{\partial x^{\prime} \partial y^{\prime}}\right)\right) \\
\epsilon \rho^{\prime} u^{\prime} \frac{\partial v^{\prime}}{\partial x^{\prime}}+\rho^{\prime} v^{\prime} \frac{\partial v^{\prime}}{\partial y^{\prime}}=-\frac{1}{\gamma M^{2}} \frac{\partial p^{\prime}}{\partial y^{\prime}}+\frac{1}{R e}\left(\epsilon^{2} \frac{\partial^{2} v^{\prime}}{\partial x^{\prime 2}}+\frac{\partial^{2} v^{\prime}}{\partial y^{\prime 2}}+\frac{1}{3}\left(\epsilon^{2} \frac{\partial^{2} v^{\prime}}{\partial y^{\prime 2}}+\epsilon \frac{\partial^{2} u^{\prime}}{\partial x^{\prime} \partial y^{\prime}}\right)\right) \\
\epsilon \rho^{\prime} u^{\prime} \frac{\partial T^{\prime}}{\partial x^{\prime}}+\rho^{\prime} v^{\prime} \frac{\partial T^{\prime}}{\partial y^{\prime}}=\epsilon \frac{\gamma-1}{\gamma} u^{\prime} \frac{\partial p^{\prime}}{\partial x^{\prime}}+\frac{\gamma-1}{\gamma} v^{\prime} \frac{\partial p^{\prime}}{\partial y^{\prime}}+\frac{1}{R e P r}\left(\epsilon^{2} \frac{\partial^{2} T^{\prime}}{\partial x^{\prime 2}}+\frac{\partial^{2} T^{\prime}}{\partial y^{\prime 2}}\right) \\
+\frac{(\gamma-1) M^{2}}{R e}\left[2\left(\frac{\partial u^{\prime}}{\partial x^{\prime}}\right)^{2} \epsilon^{2}+2\left(\frac{\partial v^{\prime}}{\partial y^{\prime}}\right)^{2}+\left(\epsilon \frac{\partial v^{\prime}}{\partial x^{\prime}}+\frac{\partial u^{\prime}}{\partial y^{\prime}}\right)^{2}-\frac{2}{3}\left(\frac{\partial u^{\prime}}{\partial x^{\prime}} \epsilon+\frac{\partial v^{\prime}}{\partial y^{\prime}}\right)^{2}\right] \\
p^{\prime}=\rho^{\prime} T^{\prime}=n^{\prime} T^{\prime}
\end{gathered}
$$

The velocity slip and temperature jump boundary conditions on the wall are, $y^{\prime}= \pm 1 / 2$ :

$$
\begin{gathered}
u_{w}^{\prime}=\mp \frac{2-\sigma_{u}}{\sigma_{u}} K n\left(x^{\prime}\right)\left(\frac{d u^{\prime}}{d y^{\prime}}\right)_{w}+\frac{3}{4} \epsilon \frac{1}{\operatorname{Re} \rho^{\prime} T_{w}^{\prime}}\left(\frac{\partial T^{\prime}}{\partial x^{\prime}}\right)_{w}=\mp \frac{2-\sigma_{u}}{\sigma_{u}} K n\left(x^{\prime}\right)\left(\frac{d u^{\prime}}{d y^{\prime}}\right)_{w} \\
T^{\prime}-T_{w}^{\prime}=\mp \frac{2-\sigma_{T}}{\sigma_{T}} \frac{2 \gamma}{\operatorname{Pr}(\gamma+1)} K n\left(x^{\prime}\right)\left(\frac{\partial T^{\prime}}{\partial y^{\prime}}\right)_{w}
\end{gathered}
$$

Note in Eqn.(18), $T^{\prime}=1+\epsilon T_{2}^{\prime}$ is used to replace the $T^{\prime}$ in the term with temperature gradient. This simplification is appropriate for both Case A and Case B.

After expanding and separating terms of different magnitudes in Eqn.(13), with the non-penetration wall condition, it is quite obvious $v_{1}=0$. Then the leading term in the y-momentum equation yields $p_{1}=f(x)$. The leading term in the $\mathrm{x}$-momentum equation yields:

$$
\frac{\epsilon R e}{\gamma M^{2}} \frac{d p_{1}}{d x^{\prime}}=\frac{\partial^{2} u_{1}}{\partial^{2} y^{\prime}}
$$

$$
5 \text { of } 14
$$


Together with the velocity slip boundary condition, the above equation yields the following result:

$$
u_{1}\left(x^{\prime}, y^{\prime}\right)=-\frac{\epsilon R e}{8 \gamma M^{2}} \frac{d p_{1}}{d x^{\prime}}\left(1-4 y^{\prime 2}+4 \Theta_{u} K n\left(x^{\prime}\right)\right)
$$

The derivation of $v_{2}\left(x^{\prime}, y^{\prime}\right)$ utilizes the continuity equation and the condition $p_{1}=\rho_{1}$, which is not obtained from the isothermal condition, instead by expanding the state equation $p_{1}+\epsilon p_{2}=\left(\rho_{1}+\epsilon \rho_{2}\right)\left(1+\epsilon T_{2}\right)$. The nontrivial continuity equation takes the following format:

$$
\frac{\partial\left(p_{1} u_{1}\right)}{\partial x^{\prime}}+\frac{\partial\left(p_{1} v_{2}\right)}{\partial y^{\prime}}=0
$$

and it yields the solution for $v_{2}$ with the aid of relation $K n_{o} / \rho_{1}\left(x^{\prime}\right)=K n\left(x^{\prime}\right)$ :

$$
v_{2}\left(x^{\prime}, y^{\prime}\right)=\frac{\epsilon R e}{8 \gamma M^{2} p_{1}}\left[\frac{1}{2} \frac{d^{2} p_{1}^{2}}{d x^{\prime 2}}\left(y^{\prime}-\frac{4}{3} y^{\prime 3}\right)+4 y^{\prime} \Theta_{u} \frac{d^{2} p_{1}}{d x^{\prime 2}} K n_{o}\right]
$$

Evaluating the above equation along the wall surface with $v_{2}=0$, yields the first order expression for pressure:

$$
p_{1}\left(x^{\prime}\right)=-6 \Theta_{u} K n_{o}+\sqrt{\left(6 \Theta_{u} K n_{o}\right)^{2}+\left(1+12 \Theta_{u} K n_{o}\right) x^{\prime}+\left(P^{2}+12 \Theta_{u} K n_{o} P\right)\left(1-x^{\prime}\right)}
$$

The above derivation procedure, from Eqn.(20) to Eqn.(24), is almost the same as Arkilic's original derivations. ${ }^{6}$ We repeat the procedure on purpose to obtain a complete set of solutions for later reference, and at the same time, to illustrate that the isothermal assumption is actually relaxed.

The next step is to obtain the temperature distribution inside the channel. After comparing the orders in Eqn.(16), the following three leading terms remain:

$$
\frac{1}{\operatorname{RePr}} \frac{\partial^{2} T_{2}}{\partial y^{\prime 2}}=-\frac{\gamma-1}{\gamma} u_{1} \frac{d p_{1}}{d x^{\prime}}-\frac{(\gamma-1) M^{2}}{\epsilon R e}\left(\frac{\partial u_{1}}{\partial y^{\prime}}\right)^{2}
$$

and the temperature jump conditions are, on $y^{\prime}= \pm 1 / 2$,

$$
1+\epsilon T_{2}-T_{w}^{\prime}=\mp \Theta_{T} \frac{2 \gamma}{\operatorname{Pr}(\gamma+1)} \epsilon K n\left(x^{\prime}\right)\left(\frac{d T_{2}}{d y^{\prime}}\right)_{w}
$$

For Case A, the three terms in Eqn.(25) hold the same order of magnitude; For Case B, the leading term in Eqn.(25) is the one on the left hand side of the equation. For both cases Eqn.(25) is solvable with the temperature jump conditions, Eqn.(26). These two cases share the exactly same temperature solution:

$$
T_{2}\left(x^{\prime}, y^{\prime}\right)=A\left(x^{\prime}\right) B\left(y^{\prime}\right)+D\left(x^{\prime}\right)
$$

where $A\left(x^{\prime}\right)=\frac{\gamma-1}{8 \gamma^{2}} \frac{\operatorname{PrR} e^{2} \epsilon}{M^{2}}\left(\frac{d p_{1}}{d x^{\prime}}\right)^{2}, B\left(y^{\prime}\right)=\left(\frac{1}{2}+2 K n\left(x^{\prime}\right) \Theta_{u}\right) y^{\prime 2}-y^{\prime 4}$, and $D\left(x^{\prime}\right)=-\frac{4 \gamma A\left(x^{\prime}\right) \Theta_{T} \Theta_{u} K n_{o}^{2}\left(x^{\prime}\right)}{\operatorname{Pr}(\gamma+1)}-$ $A\left(x^{\prime}\right)\left[\frac{1}{2} K n\left(x^{\prime}\right) \Theta_{u}+\frac{1}{16}\right]+\frac{T_{w}-1}{\epsilon}$.

The non-dimensional mass flow rate is:

$$
Q^{\prime}=\left.\int_{-1 / 2}^{1 / 2}\left(\rho_{1} u_{1}\right)\right|_{x^{\prime}=1} d y=\frac{\epsilon R e}{24 \gamma M^{2}}[P-1]\left[P+1+12 \Theta_{u} K n_{o}\right]
$$

and the dimensional mass flow rate is:

$$
Q=\left(\rho_{o} u_{o} H\right) Q^{\prime} \sim O\left(\mu \operatorname{Re}\left(P+1+12 \Theta_{u} K n_{o}\right)\right)
$$

where Eqn.(12) is used in the above equation. 
Finally we have two remarks to conclude this section:

1. This section relaxes the isothermal assumption by assuming a quasi-isothermal condition, $T^{\prime}=1+\epsilon T_{2}$, which is critical to obtain the temperature solution. This assumption is valid for microchannel flows with a slow speed and very small temperature variations, but it may be invalid for other flows in MEMS with large temperature changes, such as high speed gas flow in a microchannel with electric-magnetic field effects.

2. The obtained results are asymptotic solutions to the flow in a microchannel with two specific combinations of the Reynolds and Mach numbers. However, there may exist many other asymptotic solutions with different combinations of Mach and Reynolds numbers obeying Eqn.(12) as well. There may even exist other solutions that are only obtainable by numerical simulations.

\section{Numerical Results}

In the last section, we obtain some analytical results that require known Mach and Reynolds numbers at the microchannel exit, if we want to validate them. Although in the literature there are experimental measurements for mass flow rates and pressure distributions through particular microchannels, unfortunately, no detailed Mach and Reynolds numbers are provided. Hence, we rely on numerical simulations to provide us the needed Mach and Reynolds numbers, and then we construct analytical solutions and compare them with the numerical simulation results.

A finite volume solver of the Navier-Stokes equations is developed to perform these simulations. The inviscid and viscous fluxes across a cell edge are computed with the classical Roe scheme and a central difference scheme, respectively. The minmod function is applied in the state reconstruction stage and it provides 2nd order spatial accuracy. The slip/noslip and temperature jump/constant temperature wall boundary conditions are applied on the wall; at the microchannel exit, fixed pressure and extrapolated density are evaluated and then used to compute the corresponding temperature. Inlet, outlet, wall and symmetric boundary conditions are applied at the left, right, top and bottom sides of rectangular simulation domains.

A benchmark case is simulated to validate the Navier-Stokes equation solver, and the simulation parameters are summarized in Table 1. With known outlet Mach and Reynolds numbers, obtained from the numerical simulation, corresponding analytical solutions are constructed and compared with the numerical results.

Figure 2 shows the pressure contours, with the analytical solutions on the top and the numerical results at the bottom. It clearly shows that for this benchmark case, $d p / d y=0$, and the numerical simulation results match the analytical solutions very well. Figure 3 shows the nonlinear pressure distributions along the centerline, where the numerical and analytical results have excellent agreement.

Figure 4 shows contours for velocity along the x-direction, with the analytical results on the top and the numerical results at the bottom. As we can see, the results again agree very well. The velocity slip wall boundary conditions are used for the numerical simulation and analytical solutions, and the non-constant slip velocities on the walls are quite evident. Figure 5 shows the contours for velocity along the y-direction, with the analytical results on the top and the numerical simulation results at the bottom. On the wall and centerline, the v-velocity values are zero. The numerical and analytical results are quite close, but at the same time, for the numerical simulations, the outlet and wall boundary treatments produce significant discrepancy with the analytical solutions in these regions.

Figure 6 shows the temperature contours with the analytical solutions on the top and the numerical simulation results at the bottom. For both cases, the constant wall temperature conditions are used. This plot shows large gradients at locations close to the exit. The temperature fields have the same trends but pronounced differences, especially at the outlet. In general, with the current Navier-Stokes equation solver, it is quite challenging to obtain more accurate results for the temperature distributions. The difficulty is due to the fact that the variations in the temperature field are one order smaller than the u-velocity and the pressure fields, and the following factors account for the difference between the numerical and analytical solutions:

7 of 14

American Institute of Aeronautics and Astronautics 
1. The boundary treatment for the channel exit in the numerical simulation can result in some difference. This is also evident in Figure 5.

2. The averaged outlet Mach and Reynolds numbers, and the temperature, collected from the numerical simulation and provided to the analytical solutions, unavoidably contain some errors because of the outlet boundary condition treatment.

3. The v-velocity and temperature distributions are effected by pressure gradients. The pressure contours already contain some difference, see Figure 2, and the pressure gradients from the two solutions must be huge due to the small microchannel length. The temperature contours are more sensitive as they contain a term in $(d p / d x)^{2}$. Hence, poorer agreement is not surprising for temperature.

4. The analytical solution for the temperature is actually a leading term, while numerical simulation results contain the whole solution.

To obtain better results for the temperature distribution is a challenging task and it requires better treatment of the outlet boundary conditions.

In general, comparisons of the results for this benchmark case show that the Navier-Stokes equation solver is reliable to provide the correct results for the pressure and velocities in the $\mathrm{x}$ - and $\mathrm{y}$ - directions. Meanwhile, it does provide temperature distributions with correct trends.

Figure 8 shows the normalized pressure distributions along the channel centerline, with numerical simulation results, analytical results, and the experimental measurements by Pong et al. ${ }^{4}$ To further clearly display the nonlinear deviation, Figure 9 shows the corresponding values $\left(p-p_{\text {linear }}\right) /\left(p_{i}-p_{0}\right)$. For this case, the parameters are, $H=1.2 \mu \mathrm{m}, L=36 \mu \mathrm{m}, P_{o}=1 \mathrm{~atm}, P=1.891$, nitrogen gas, $\sigma_{u}=0.85$ and constant wall boundary conditions. This is a test case from Group 1 . It is very clear that the simulation and analytical results are very close to the experimental measurements in Figure 8, and they almost bound the measurements results in Figure 9.

To illustrate the temperature jump effects on the wall, Figure 7 shows two analytical solutions with the same Mach and Reynolds numbers at the exit. For the case on the top, wall boundary conditions of velocity slip and temperature jump with $\sigma_{T}=0.85$ are used, while for the case at the bottom, wall boundary conditions of velocity slip and constant temperature are used. This picture shows the same trends for temperature distributions, but the temperature jump wall boundary condition results in much larger variations and lower temperatures at the outlet.

With the validity of the Navier-Stokes equation solver properly examined, we further perform several simulations to validate the Reynolds-Mach number relation, Eqn. (12), and the mass flow rate relation, Eqn. (29). The simulation parameters are summarized in Table 1 as well.

As one of the major results from this study, we want to confirm the relation between the Mach number and the Reynolds number at the channel exit, i.e, Eqn.(12), for the situations of slip wall boundary conditions or nonslip wall boundary conditions. Figure 10 shows the Reynolds and Mach numbers at the exits for the cases in Group 1. Figure 11 shows the corresponding Reynolds and Mach number variations at channel exits for the cases in Group 2. Each symbol in these two pictures actually represents one simulation for a microchannel with different combination of parameters, such as different channel length, pressure or wall boundary conditions. Both plots show that the slip flow cases have larger Reynolds and Mach numbers, due to larger exit velocities. The Mach numbers are certain to be smaller than the corresponding Reynolds numbers for these given test cases. There are two ways to interpret the results: first, the Reynolds and Mach numbers vary almost linearly as $\epsilon$ increases, hence the relations $R e=O(\epsilon)$ and $M a=O(\epsilon)$ for Case B hold; on the other hand, if we examine the actual values for the Reynolds and Mach numbers, these two plots show that the values of $R e$ are on the order of unity, and the values of $M a$ are on the order of $\epsilon^{1 / 2}$. Hence, the cases from these two groups qualify for Cases A as well. When we consider the fact that the Reynolds and Mach numbers are used to normalize the Navier-Stokes equations, it is more appropriate to conclude that these results fall in the range of Case A rather than that for Case B. Figure 12 shows the 
relation among the pressure ratios, $P$, the channel dimension ratio, $\epsilon$, and the Reynolds/Mach numbers at the channel exits. It does show clear linear relations as predicted by Eqn.(12). Two straight lines are added to aid the comparison.

The next three figures show dimensional mass flow rates vs. several parameters. Figure 13 shows a weak nonlinear relation between the mass flow rate and the inlet/outlet pressure ratio, $P$. Figure 14 shows the linear relation between the mass flow rate and the channel dimension ratio, $\epsilon$, with fixed pressure ratio, $P=1.891$. Figure 15 shows the relation between the mass flow rate and $\operatorname{Re}\left(P+1+12 \Theta_{u} K n_{0}\right)$ for the slip flow situation or $\operatorname{Re}(P+1)$ for the nonslip flow situation. With two clear linear relations observed, this plot validates Eqn.(29).

\section{Conclusion}

This paper extended Arkilic's original work on analytically studying the compressible gas flow field inside a two-dimensional microchannel. In summary, the following improvement was obtained:

1. By comparing the different forces inside a microchannel, i.e., the viscous force, the pressure drop and the momentum change, we obtained a fundamental relation, Eqn.(12), which links the Mach and Reynolds numbers at the channel exit, the pressure drop ratio, $P$, and the channel aspect ratio, $\epsilon$. It illustrated that Arkilic's estimation for the Mach number and Reynolds number was one special case from this relation. This important relation provides a guideline to construct different orders of Reynolds and Mach numbers in future studies.

2. By including the energy equation, and dropping the isothermal assumption, which was used by Arkilic and several other researchers, a detailed temperature field described by Eqn.(27) was obtained. The quasi-isothermal condition is applicable to low speed compressible flows inside microchannels and it is crucial to obtained the temperature solution.

3. The dimensional mass flow rate essentially follows Eqn.(29) and Figure 15 confirmed this conclusion. The pressure ratio has a weak nonlinear effect on the mass flow rate since it has effects on the Reynolds number as well.

4. We validated the analytical results by series of numerical simulations performed with a Navier-Stokes equation solver. However, the temperature distribution is the most difficult property to capture due to several reasons we summarized.

\section{Acknowledgments}

This work was motivated and partially completed at the University of Michigan, while the first author was concluding his doctoral study.

\section{References}

${ }^{1}$ Prud'homme, R.K., Chapman, T.W. and Brown, J.R., "Laminar Compressible Flow in a Tube," Appl. Sci. Res., 1986, 43: pp.67-74.

${ }^{2}$ Van Den Berg, H.R., Seldam, C.A. and Van der Guli, P.S., "Compressible Laminar Flow in a Capillary," Journal of Fluid Mechanics, 1993, Vol.246, pp.1-20. 274 .

${ }^{3}$ Harley, J.C. and Huang, Y.H., et. al., "Gas Flow in Micro-channels," Journal of Fluid Mechanics, 1995, vol.284, pp.257-

${ }^{4}$ Pong, K. C., Ho, C. M. and Liu, J. Q., et al, "Nonlinear Pressure Distribution in Uniform Microchannels," $A S M E-F E D$ 197, pp.51-56, 1994.

${ }^{5}$ Arkilic, E.B., "Measurement of the Mass Flow and Tangential Momentum Accomodation Coefficient in Silicon Microchannel," Ph.D. Dissertation, Department of Aeronautical and Aerospace Engineering, Massachusate Institute of Technology, 1994.

9 of 14

American Institute of Aeronautics and Astronautics 
${ }^{6}$ Arkilic, E.B., Schmidt, M.A. and Breuer, K.S., "Gaseous Slip Flow in Long Microchannels," Journal of Microelectromechanical Systems, Vol. 6, No. 2, June 1997, pp.167-178.

${ }^{7}$ Karniadakis, G.E. and Beskok, A., Microflows: Fundamentals and Simulation., 2002, Springer.

${ }^{8}$ Zohar, Y. Lee, S. Y. K. Lee, W.Y., Jiang, L. and Tong, P., "Subsonic Gas Flow in a Straight and Uniform Microchannel," Journal of Fluid Mechanics, Vol.472, 2004, pp.125-151.

${ }^{9}$ Ho, C.M. and Tai, Y.C., "Micro-Electro-Mechanical-Systems(MEMS) and Fluid Flows," Annual Review of Fluid Mechanics, Vol.30, 1998, pp.579-612.

${ }^{10}$ Oran, E.S., Oh, C.K. and Cybyk, B.Z., "Direct Simulation Monte Carlo: Recent Advances and Applications," Annual Review of Fluid Mechanics, Vol.30, 1998, pp.403-441.

${ }^{11}$ Zheng, Y., Garcia, A. L. and Alder, B.J., "Comparison of Kinetic Theory and Hydrodynamics for Poiseuille Flow," Rarefied Gas Dynamics, Vol.23, Whislter Canada, 2002.

${ }^{12}$ Cai, C., Boyd, I., Fan, J. and Candler, G. V., "Direct Simulation Methods For Low-Speed Microchannel Flows," Journal of Thermophysics and Heat Transfer, Vol.14, July-September, 2000, pp.368-378.

${ }^{13}$ Aristov, V.V., Direct Methods for Solving the Boltzmann Equations and Study of Nonequilibrium Flows, Kluwer, 2001.

${ }^{14}$ Ohwada, T., Sone, Y. and Aoki, K., "Numerical Analysis of the Poiseuille Flow and Thermal Transpiration Flows between Two Parallel Plates on the Basis of the Linearized Boltzmann Equation for Hard-Sphere Molecules," Physics of Fluids, Vol.1, pp.2042, 1989.

${ }^{15} \mathrm{Xu}, \mathrm{K}$. and Li, Z., "Microchannel Flow in the Slip Regime: Gas-Kinetic BGK-Burnett Solutions," Journal of Fluid Mechanics, Vol.513, pp.87-110, 2004.

${ }^{16}$ Chen, C.K. and Weng, H.C., "Developing Natural Convection with Thermal Creep in a Vertical Microchannel," Journal of Physics D: Applied Physics, Vol.39, pp.3107-3118, 2006.

${ }^{17}$ Du, D.X., Li, Z.X. and Guo, Z.Y., "Effect of Reversible Work and Viscous Dissipation on Gas Flow Characteristics in a Microtube," Journal of TshingHua University Science and Technology, Vol.39, No.11, 1999.

${ }^{18}$ White, F. M., Vicous Fluid Flow, McGraw-Hill, 1991.

${ }^{19}$ Chen, X., GasKinetics and Its Applications in Heat Transfer and Flow, TsingHua University Press, Beijing, 1996.

\begin{tabular}{|l|r|r|r|}
\hline & Benchmark Case A & Group 1 & Group 2 \\
\hline Gas & $O_{2}$ & $N_{2}$ & $N_{2}$ \\
\hline $\mathrm{L}, \mu m$ & 15 & $36,72,108,144,216$ & 72 \\
\hline $\mathrm{H}, \mu m$ & 0.53 & 1.2 & 1.2 \\
\hline Inlet Pressure, $10^{5} \mathrm{~Pa}$ & 2.5 & 1.891 & $1.891,2.5,3.0,3.5,4.0$ \\
\hline Outlet Pressure, $10^{5} \mathrm{~Pa}$ & 1.0 & 1.0 & 1.0 \\
\hline$T_{w}, T_{i}, K$ & 300 & 300 & 300 \\
\hline cell number & $200 \times 67$ & $250 \times 67$ & $250 \times 67$ \\
\hline viscosity index & 0.77 & 0.70 & 0.70 \\
\hline$\sigma_{u}$ & nonslip, or 0.85 & nonslip, or 0.85 & nonslip, or 0.85 \\
\hline$\sigma_{T}$ & const temp & const temp, or 0.85 & const temp, or 0.85 \\
\hline
\end{tabular}

Table 1. Computation Conditions.

10 of 14

American Institute of Aeronautics and Astronautics 


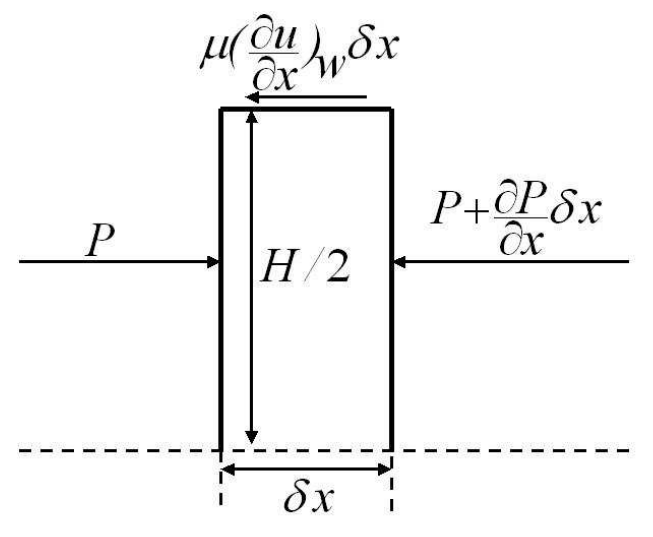

Figure 1. Illustration of friction force, pressure drop, and momentum change in a microchannel.

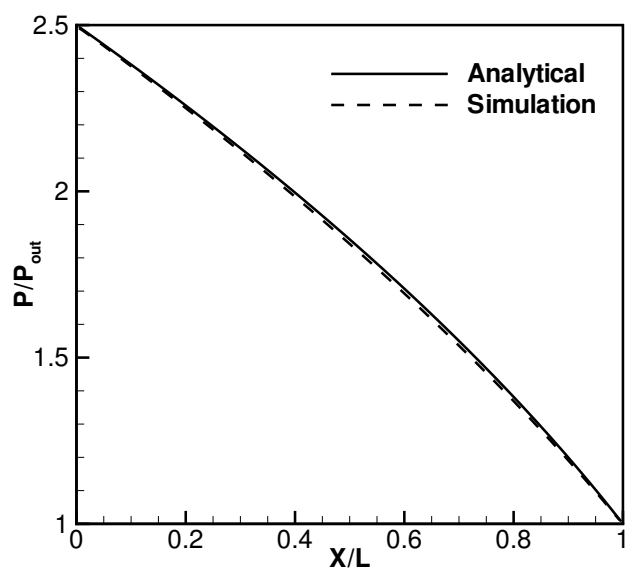

Figure 3. Comparison of centerline pressure distributions for the benchmark case.

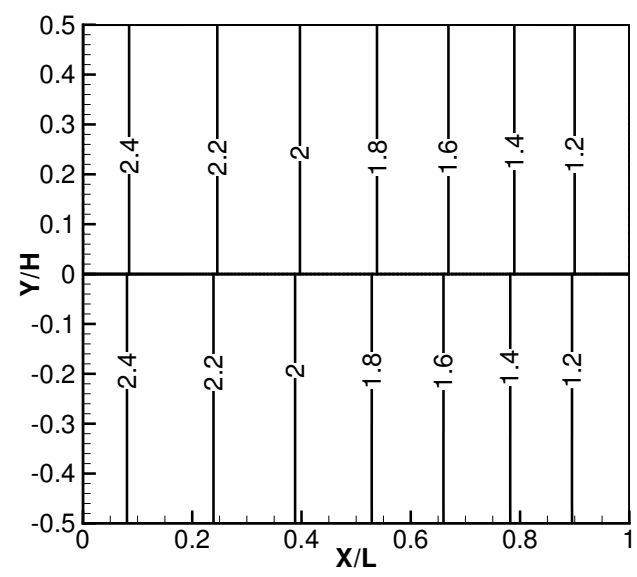

Figure 2. Comparison of pressure contours for the benchmark case. Top: analytical, bottom: numerical simulation.

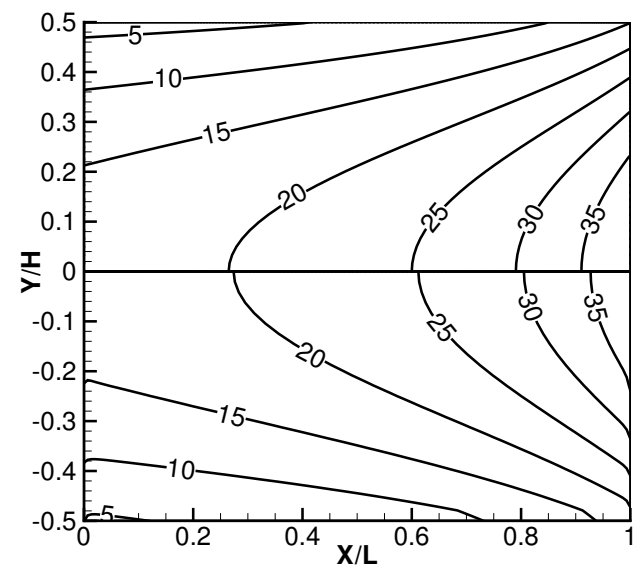

Figure 4. Comparison of $x$-velocity contours from analytical solutions(top) and numerical simulation(bottom), the benchmark case. 


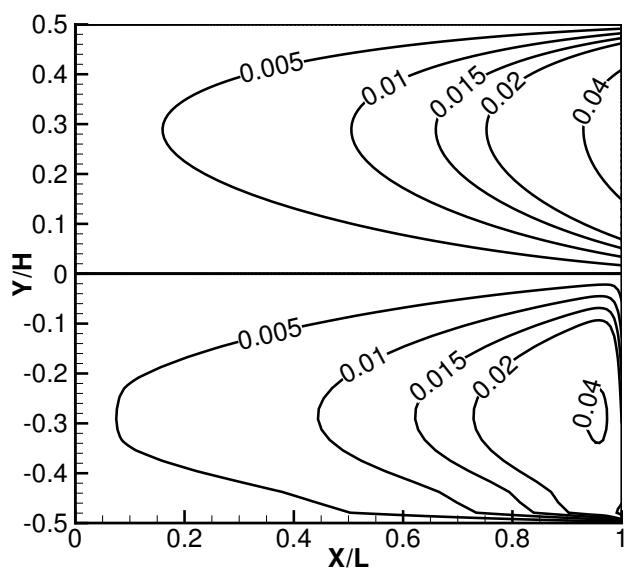

Figure 5. Comparison of y-velocity contours from analytical solutions(top) and numerical simulation(bottom), the benchmark case.

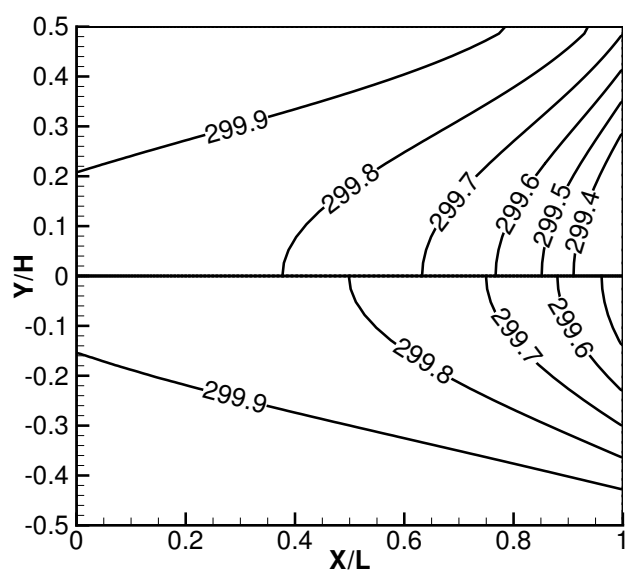

Figure 7. Comparison of temperature contours from the analytical solutions. Top: $\sigma_{T}=0.85$, bottom: constant wall temperature, the benchmark case.

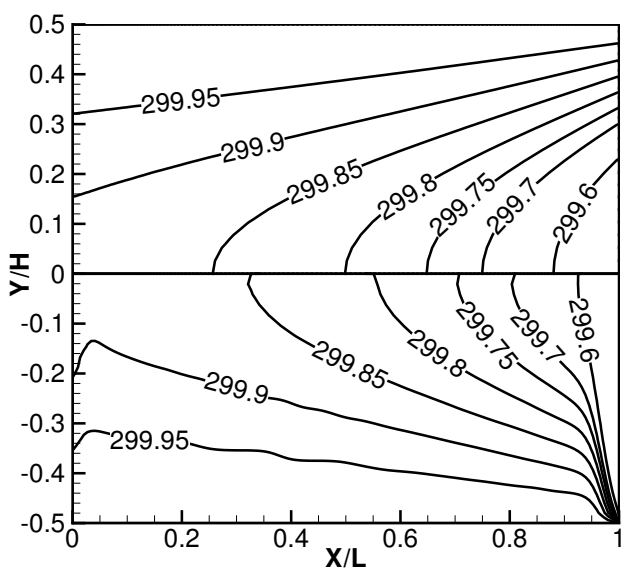

Figure 6. Comparison of temperature contours from analytical solutions(top) and numerical simulation(bottom), the benchmark case.

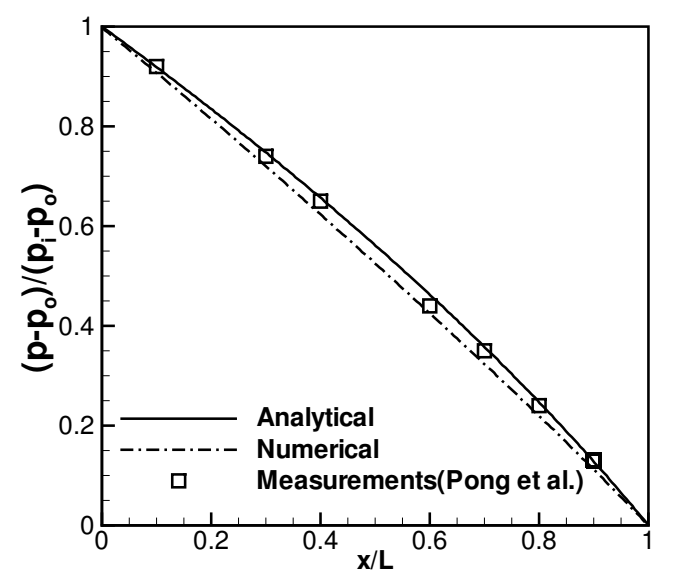

Figure 8. Normalized pressure distribution for nitrogen gas in a channel with $H=1.2 \mu \mathrm{m}, L=36$ $\mu m, P=1.891$ and $P_{o}=1 \mathrm{~atm}$. 


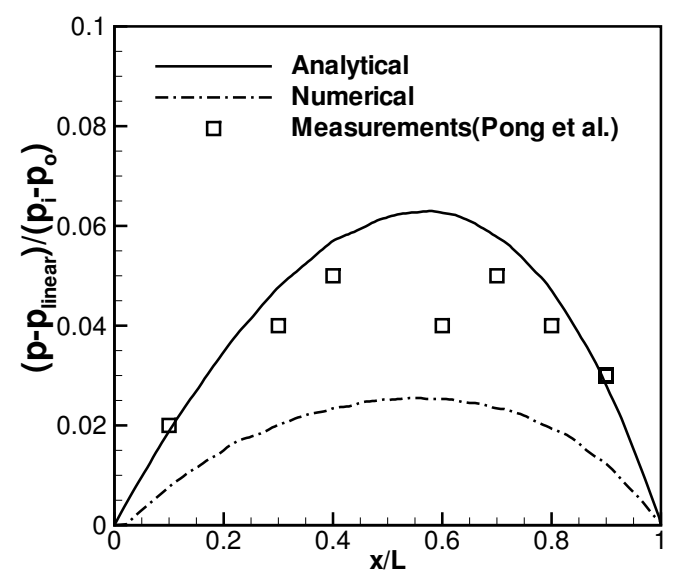

Figure 9. Pressure distribution nonlinearity for nitrogen gas in a channel with $H=1.2 \mu \mathrm{m}, L=36$ $\mu m, P=1.891$ and $P_{o}=1 \mathrm{~atm}$.

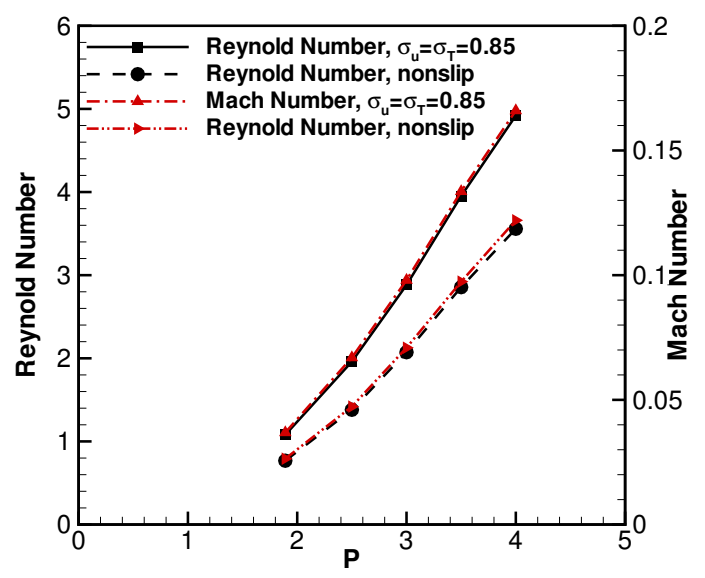

Figure 11. Variations in outlet Reynolds and Mach numbers, Group 2 results, $H=1.2 \mu \mathrm{m}$, $L=72 \mu m$,

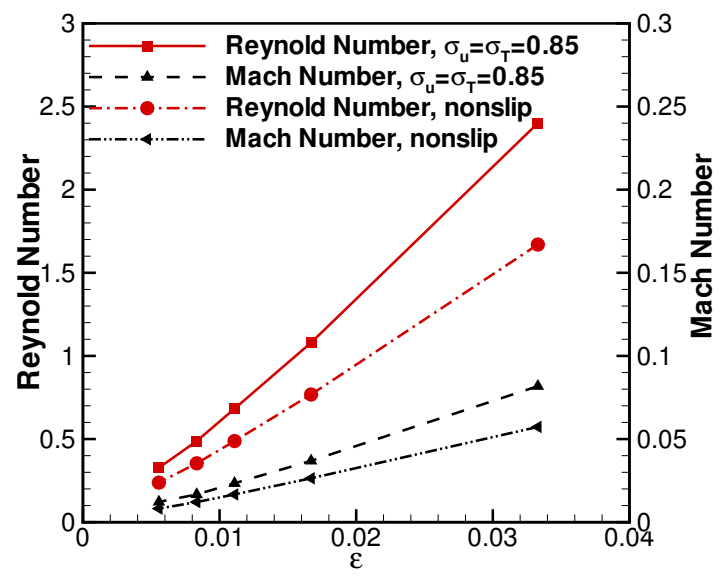

Figure 10. Variations in outlet Reynolds and Mach numbers, Group 1 results, $H=1.2 \mu \mathrm{m}$, $P=1.891$.

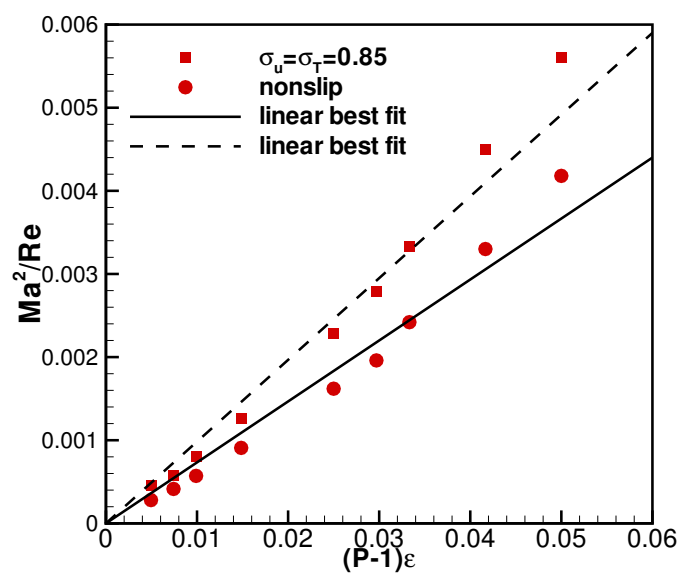

Figure 12. Relations between $M a^{2} / R e$ and $(P-1) \epsilon$ at channel exits, test cases in Groups 1 and 2. 


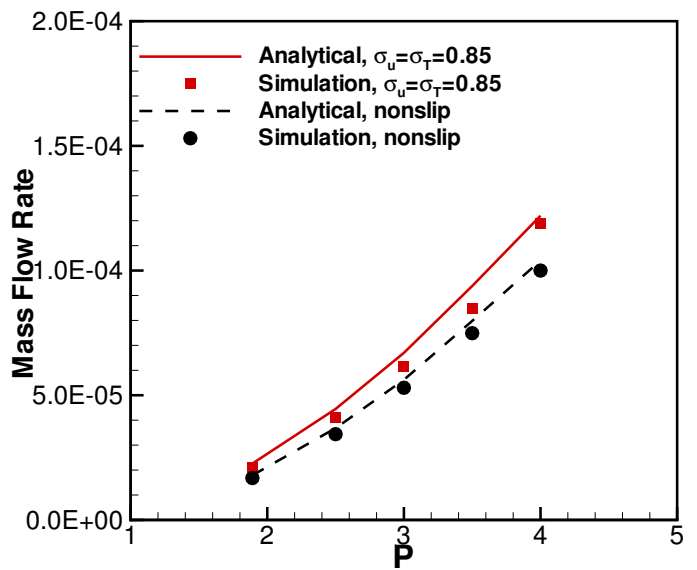

Figure 13. Mass flow rate vs. different pressure ratios, $H=1.2 \mu m$ and $L=72 \mu m$.

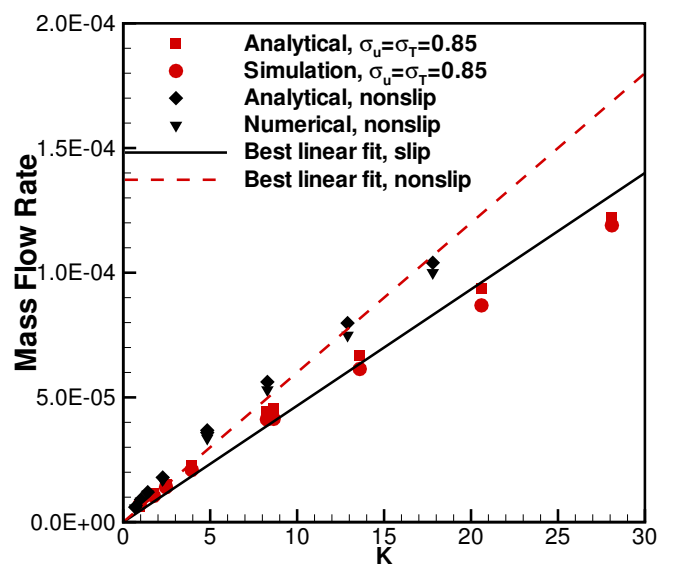

Figure 15. Dimensional mass flow rate vs. K, where $K=\operatorname{Re}\left(P+1+12 \Theta_{u} K n_{o}\right)$ for slip flows and $K=\operatorname{Re}(P+1)$ for nonslip flows.

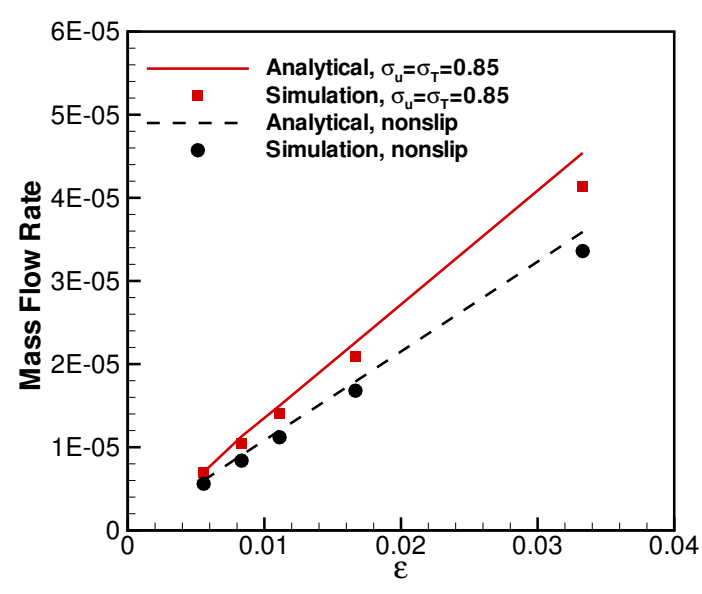

Figure 14. Mass flow rate vs. dimension ratios, $H=1.2 \mu m$ and $P=1.891$. 